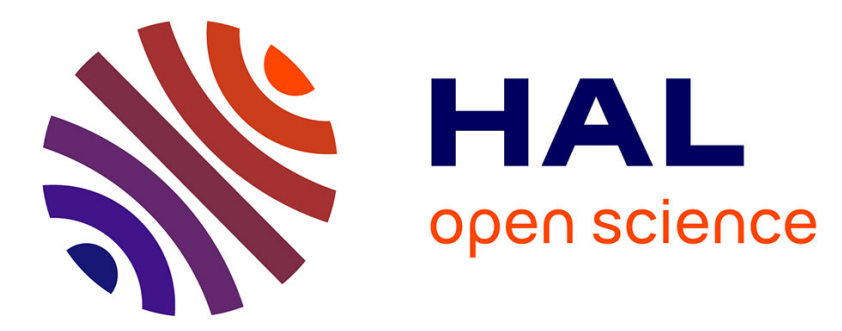

\title{
Drying kinetics and acoustic properties of soft porous polymer materials
}

Raj Kumar, Y. Jin, Samuel Marre, Olivier Poncelet, Thomas Brunet, Jacques Leng, Olivier Mondain-Monval

\section{To cite this version:}

Raj Kumar, Y. Jin, Samuel Marre, Olivier Poncelet, Thomas Brunet, et al.. Drying kinetics and acoustic properties of soft porous polymer materials. Journal of Porous Materials, 2020, 28 (1), pp.249-259. 10.1007/s10934-020-00987-w . hal-02975532

\section{HAL Id: hal-02975532 \\ https://hal.science/hal-02975532}

Submitted on 26 Oct 2020

HAL is a multi-disciplinary open access archive for the deposit and dissemination of scientific research documents, whether they are published or not. The documents may come from teaching and research institutions in France or abroad, or from public or private research centers.
L'archive ouverte pluridisciplinaire HAL, est destinée au dépôt et à la diffusion de documents scientifiques de niveau recherche, publiés ou non, émanant des établissements d'enseignement et de recherche français ou étrangers, des laboratoires publics ou privés. 


\title{
Drying kinetics and acoustic properties of soft porous polymer materials
}

\author{
R. Kumar ${ }^{1}$, Y. Jin ${ }^{2,+}$, S. Marre ${ }^{3}$, O. Poncelet ${ }^{2}$, T. Brunet ${ }^{2}$, J. Leng ${ }^{4}$, O. Mondain-Monval ${ }^{1}$ \\ ${ }^{1}$ Univ. Bordeaux, CNRS, UMR 5031 CRPP, F-33600 Pessac, FRANCE \\ ${ }^{2}$ Univ. Bordeaux, CNRS, Bordeaux INP, ENSAM, UMR 5295 I2M, F-33405 Talence, FRANCE \\ ${ }^{3}$ Univ. Bordeaux, CNRS, Bordeaux INP, UMR 5026 ICMCB, F-33600, Pessac, France \\ ${ }^{4}$ Univ. Bordeaux, CNRS-Solvay, UMR 5258 LOF, F-33600 Pessac, France \\ 'Present Address: School of Aerospace Engineering and Applied Mechanics, Tongji University, CHINA
}

\begin{abstract}
We describe a method for the fabrication of acoustic metasurfaces, which is based on soft porous polymer materials. The materials are obtained using an emulsion templating technique, which allows for the fabrication of soft porous polymers with fully controlled porosity values between 0 and 30\%. Our approach involves the polymerization of water-in-silicone emulsions with controlled water volume fractions. The obtained wet solid monolith samples are dried using three different methods. Due to the softness of the polymer matrix such as, polyHIPE hydrogels or like in silica aerogels, the first method regular air drying - leads to a collapse of the material and we present a complete experimental study of the observed kinetics as well as a model to account for the observed results. We show that this model can catch the kinetics characteristics. Then, using two alternative drying techniques, $\mathrm{H}_{2} \mathrm{O}_{2}$-assisted and supercritical drying, we show that it is possible to obtain materials with fully controlled porosities. The speed of sound - or equivalently the material acoustic index - inside the material being dependent on its porosity, we obtain a gradient-index acoustic material by spatially controlling the porosity distribution along the two dimensions of these metasurfaces. Their ability in terms of wavefront shaping is then demonstrated through a deflecting experiment performed in water with a sample having a thickness 5 times smaller than the incident acoustic wavelength at ultrasonic frequencies.
\end{abstract}




\section{Introduction}

Metasurfaces are 2D materials of sub wavelength thickness that can provide non-trivial local phase shifts or amplitude modulation (or gradient) of the waves. Such devices enable a full spatial control of the wavefront. For example, a metasurface with appropriate properties can deflect or focus a beam. In other cases, it may also be used to produce twisted beams [1]. Initially introduced for electromagnetic waves, the concept has been extended to acoustics [2] and other areas of wave physics. Following those tracks, acoustic gradient index (GRIN) metasurfaces, i.e. surfaces in which the acoustic index varies as a function of position in the material, have been the object of intense research in the last years, leading to many realizations, mainly for air-borne acoustic waves [2-6]. The surfaces are obtained by micromechanical approaches, e.g. 3D printing techniques, and are based on the use of labyrinthine structures (with sizes on the order of $1 \mathrm{~cm}$ ) allowing for a fine control of the wavefront in air at audible frequencies. Unfortunately, this smart labyrinthine approach cannot be used in water owing to the much larger acoustic impedance of water with respect to air [7]. Therefore, only few devices working in a very narrow frequency range [8] have been produced for water-borne acoustics, a domain of great interest for underwater applications and medical ultrasound devices. In a recent paper [9], we demonstrated that very thin sheets composed of soft porous polymers can be used as acoustic metasurfaces for water-borne ultrasonic waves, which paves the way to the realization of many acoustic devices working in fluid phases. However, as previously observed, the fabrication of homogeneous soft porous polymer materials is difficult due to the collapse of the structure upon drying [10], a problem also encountered for the synthesis of silica aerogels [11], polyHIPEs [12] or soft hydrogels [13]. In this paper, we describe the experimental procedure allowing for the production of broadband acoustic GRIN metasurfaces made of soft porous materials achieved using an emulsion templating technique (the so-called polyHIPEs approach $[14,15])$ and specifically adapted drying techniques. We focus on the understanding of the drying mechanism and on the structural properties of the obtained samples. We adapt two drying techniques, which 
allows to produce soft samples with fully controlled porosity between 0 and $30 \%$. Then, we show one examples of a device capable of manipulating sound waves with a thickness much smaller than the wavelength $\lambda_{0}$ (typically $\lambda_{0} / 5$ ).

\section{Synthesis of samples}

The wet macroporous monolith samples were obtained by the UV polymerization of a water-in-Poly(dimethyl siloxane) (PDMS) emulsion as previously reported by our group $[16,17]$. The continuous phase of the emulsions was obtained by mixing $12.45 \mathrm{~g}$ of silicone-based UV cross-linkable PDMS fluid (Silcolease UV Poly200 ${ }^{\text {тM }}$ from Bluestar Silicones) together with $90 \mathrm{mg}$ of catalyzer (Silcolease UV Cata211 ${ }^{\text {TM }}$ from Bluestar Silicones), $10 \mathrm{mg}$ of UV sensitizer (2-isopropylthioxanthone from Rahn AG) and $50 \mathrm{mg}$ of surfactant (2-octyl-1-dodecanol from Aldrich). In case of regular and supercritical drying, water phase containing $1.5 \mathrm{wt} . \% \mathrm{NaCl}$ was introduced under mechanical stirring with a home-made helical device. The size distributions of emulsions were characterized by static light scattering using a Mastersizer S apparatus (Malvern) and optical microscopy (Leica DM 2500P microscope). The emulsions were introduced into Teflon (PTFE) molds of various thicknesses and maintained between two quartz plates, to avoid water evaporation during the polymerization process and achieve samples with smooth surfaces. UV was applied for typically $5 \mathrm{~min}$ to each faces of the samples. For $\mathrm{H}_{2} \mathrm{O}_{2}$-assisted drying, the dispersed phase included various proportion of $\mathrm{H}_{2} \mathrm{O}_{2}$.

\section{Drying of samples}

\subsection{Regular drying}

In a recent study [10], we showed that the drying of porous samples could lead to collapse phenomena due to the negative pressure that takes place inside water droplets as the liquid leaks out of the materials during the drying step. Such collapse was only observed in systems where the inside water droplets do not stick to each other before the polymerization. In such case, the obtained polymerized materials is of the closed cell type, with no direct 
connections between the pores containing the water. In this case, the permeation of the water from the inside of the pores to the exterior occurs through the polymer walls and the materials collapse during drying. As we showed before, such a collapse is not observed when the porosity of the sample is connected through micrometric holes appearing between the pores during the polymerization process. One possible way to interpret this phenomenon is to consider that during the drying, the sample is submitted to a drying pressure:

$$
P_{\text {drying }}=2 \gamma / R
$$

where $\gamma$ is the surface tension of water and $R$ the typical size of the channels by which the liquid is permeating through the matrix. For connected droplets such as the ones obtained with aggregated emulsions, one gets $R \sim 1 \mu \mathrm{m}$ and $P_{\text {drying }} \sim 0.1 \mathrm{MPa}$. If the droplets are isolated in the material, water is permeating through the sample and the characteristic size is almost molecular and typically smaller than $1 \mathrm{~nm}$, so $P_{\text {drying }} \sim 100 \mathrm{MPa}$. These values should be compared to the Young's modulus $E_{0}$ of the PDMS matrix ( $7 \mathrm{MPa}$ in our case) and explains why the pores collapse

in the system with a closed porosity $\left(P_{\text {drying }} \gg E_{0}\right)$ but not with the opened one $\left(P_{\text {drying }} \ll<E_{0}\right)$. To avoid this phenomenon, we identified two different methods that will be presented below.

First, to further understand the underlying mechanisms of drying, we performed a complete experimental study of the drying kinetics and we propose a model to account for the obtained results.

\subsubsection{Drying kinetics measurements}

First, we examine the spontaneous drying kinetics of monoliths left in an oven at a given temperature $\left(60^{\circ} \mathrm{C}\right.$, constant humidity). The monoliths have the shape of disks with diameter $32 \mathrm{~mm}$ and thicknesses varying between 1 and $5 \mathrm{~mm}$. In order to simplify the geometry of drying, the edges of the disks were covered by a Teflon tape while their faces were freely exposed to the ambient hot air. The drying kinetics was acquired by regularly weighing the 
samples over time for a set of samples with varying water volume fraction ( 2 to $30 \%$ ). To quantify the drying rate, we express the relative mass loss of water $W$ :

$$
W(t)=\frac{m(t)-m(\infty)}{m(0)-m(\infty)}
$$

where $m(0), m(t), m(\infty)$ stand for the mass of the samples at times $0, t$, and infinity respectively. We also checked that the hygrometry of the oven is not altered by the release of water during drying by evaporating a small volume of pure water $(\approx 2 \mathrm{~mL})$ also regularly weighted over time; it leads to the linear decrease of the mass (data not shown) which is consistent, we believe, with a constant humidity.

Fig. 1 collects the two main sets of data concerning the drying kinetics: First (Fig. 1, top left) a drying kinetics at constant slab thickness and varying initial volume fraction $\phi$ of water; second (Fig. 1, bottom left) a drying kinetics at constant volume fraction but varying slab thickness. 

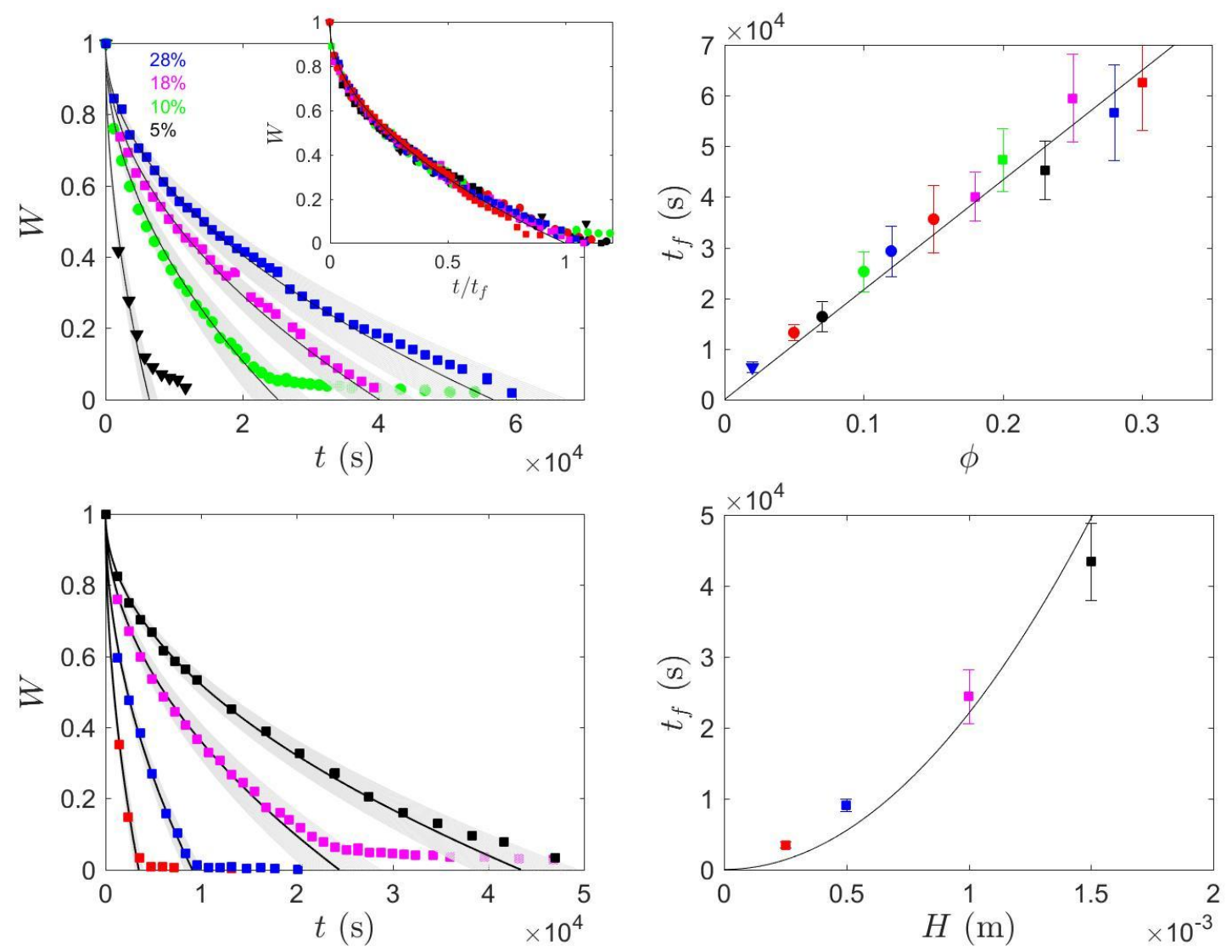

Figure 1: (Top Left) Example of relative mass loss against time for four different initial volume fractions of water dispersed in the silicone (slab of thickness $2 H=2 \mathrm{~mm}$ ); the solid line is a fit according to the model $W=1-\left(t / t_{f}\right)^{1 / 2}$, see text (eq. (3)), from which we extract the final drying time $t_{f}$ at which $W=0$. The shaded zones indicate the error on the fit, i.e., on $t_{f}$. (Insert). All eleven drying kinetics are expressed against the reduced time $t / t_{f}$, the solid line being $W=1-\left(t / t_{f}\right)^{1 / 2}$ (volume fractions: $210^{-2}, 510^{-2}, 710^{-2}, 10^{-1}, 1210^{-2}, 1510^{-}$ ${ }^{2}, 1810^{-2}, 2010^{-2}, 2310^{-2}, 2510^{-2}, 3010^{-2}$ ). (Top Right) Final drying time against the volume fraction of dispersed water in silicone and a linear fit $t_{f}=\alpha \phi$ with $\alpha=(2.2 \pm 0.1) 10^{5} \mathrm{~s}$. (Bottom Left) Relative water mass loss $W$ against time for four different thicknesses of the slab (volume fraction of dispersed water $\phi=0.3$ ); (Bottom Right) Final drying time versus thickness of the slab where the line is a fit

$$
t_{f}=\beta H^{2} \text { with } \beta=(22 \pm 4) 10^{9} \text { s. m }{ }^{-2} \text {. }
$$

In all cases, the relative mass loss $W$ fits reasonably well a simple law $W=1-a t^{1 / 2}$, or equivalently:

$$
W=1-\left(t / t_{f}\right)^{1 / 2}
$$


where the final drying time $t_{f}$ is defined as $W\left(t_{f}\right)=0$. Experiments suggest that $t_{f} \propto \phi H^{2}$, see Fig. 1, which we will interpret below with a simple model.

To follow further the drying of the materials, we performed Scanning Electron Microscopy (SEM) observations (Hitachi TM-1000) of samples sacrificed during the course of drying: as time passes, we cut several slabs and observe their side-views. Interestingly, we observe the progression of a front that separates a zone near the outer surface where the porosity has collapsed and an inner zone with preserved porosity, see Fig. 2 . Also interesting is the shape of the collapsed pores after drying, which are more visible on Fig. 2 (Bottom) and can be compared to the one observed by Milner et al. [18]. These authors studied the behavior of an individual water droplet initially stuck into PDMS matrices of various softness. As the water leaks out of the internal pore through the matrix, they observe various types of deformation of the PDMS material depending on the toughness of the matrix. In "tough" matrices (i.e. in their case, as the polymer shear modulus exceeds around $200 \mathrm{kPa}$ ), they observe a deformation of the pore that is very similar to the one observed in our sample (see the Fig. 3 of their paper). In our case, the shear modulus was measured to be $\approx 3 \mathrm{MPa}(\gg 200 \mathrm{kPa})$ and we do observe the same kind of deformation. 

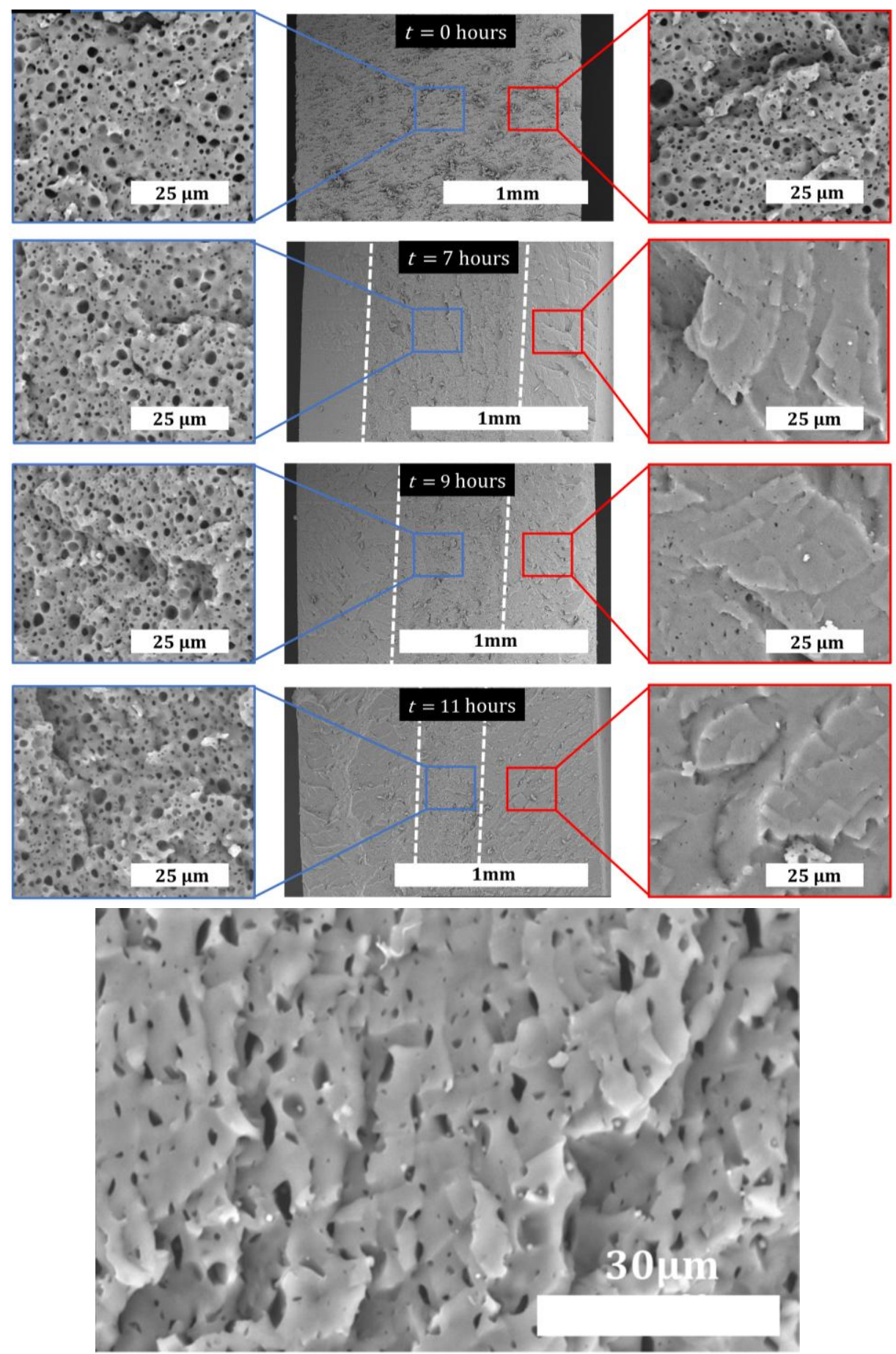

Figure 2: (Top) SEM side-views pictures of slabs with thickness $2 \mathrm{~mm}$ and $\phi=0.3$ upon drying; From top to bottom: initial $\mathrm{material}$ at $t=0$; $t=7 \mathrm{~h} ; t=9 \mathrm{~h}$ and $t=11 \mathrm{~h}$. Pictures on left and right are the zoom of the red and blue zones respectively. (Bottom) Zoom picture of the dried zone evidencing a peculiar shape for the collapsed pores. 


\subsubsection{Drying kinetics model}

We now derive a very basic view of what is likely to happen during drying. In brief, the slab containing some volume fraction $\phi$ of dispersed water dries because the water permeates from the embedded drops to the surface of the slab in contact with an unsaturated atmosphere: the difference of chemical potential of water inside and outside the slab is the driving force of the permeation process. Yet, the kinetics is extremely complex because it involves describing the concentration field of water solubilized in the silicone everywhere in between the drop, in space and time, with droplets behaving as water source up to a point they cavitate and collapse. It is clearly beyond our goal and we circumvent such a hard problem with simplifying assumptions.

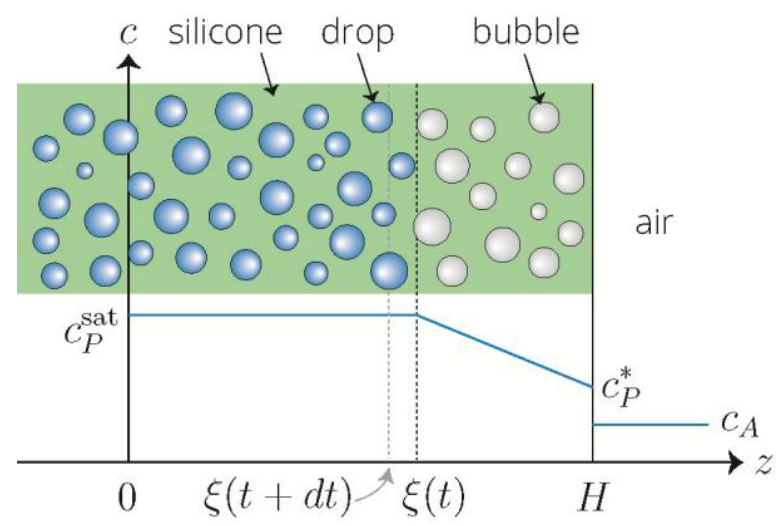

Figure 3: Sketch of the model: side view of the slab, symmetrical with respect to the plane $z=0$ and initially filled with a water emulsion at volume fraction $\phi$. Due to the permeation of water across the silicone to the interface in contact with unsaturated air, the drops empty up with time. We assume that there is a drying front at a position $\xi(t)$ before which all drops are empty (dry zone) and after which they remain full (wet zone).

First, we define in Fig. 3 the geometry of the slab by its thickness $2 H$ and assume the diffusion problem reduces to a one-dimensional problem along the $z$ dimension perpendicular to the faces of the slab. Then, we assume that the boundary condition $(\mathrm{BC})$ at the surface of the slab is of fixed concentration of water in the polymer $c_{P}^{*}$, in local 
equilibrium with the outer concentration $c_{A}$ of water in air. In principle, we should set a flux BC but basic estimates evidence that the mass transport of water is strongly limited by diffusion inside the slab, not outside, therefore justifying a concentration BC. Then, we assume that everywhere deep in the sample-in the wet zone, see Fig. 3the silicone is fully saturated with solubilized water at concentration $c_{P}^{\text {sat }}$ provided by water droplets acting as sources; near the interface however, the permeation-induced drying process empties the drops. We therefore split the slab into a dry and a wet zone with a well-defined front, as indeed observed experimentally, see Fig. 2, and also confirmed by finite element calculations (not shown here). Consequently, the concentration field in the dry zone falls (about) linearly from saturation to surface concentration, and induces a drying flux:

$$
j_{d}=-D_{P}\left(c_{P}^{\mathrm{sat}}-c_{P}^{*}\right) /(H-\xi)
$$

with $D_{P}$ the diffusion coefficient of water in the silicone $\left(\approx 10^{-9} \mathrm{~m}^{2} \mathrm{~s}^{-1}\right.$ at room temperature).

Now, we assume that the drying front evolves slowly (justified below), which sets a quasi-static model for the mass conservation and provides a kinetic equation for the propagation of the front. The latter moves because the drying flux pumps the water, the source being the drop which deliver a molar mass $-\phi / \tilde{v} \frac{d(H-\xi)}{d t}$ where $\phi / \tilde{v}$ is an equivalent concentration ( $\tilde{v}$ is the molar volume of liquid water, i.e., the ratio of molar mass and density of water: $\left.\tilde{v}=\frac{M_{w}}{\rho_{w}} \approx 1810^{-6} \mathrm{~m}^{3} \cdot \mathrm{mol}^{-1}\right)$

Equating these two fluxes yields:

$$
[H-\xi(t)]^{2}=\left[2 D_{P}\left(c_{P}^{\mathrm{sat}}-c_{P}^{*}\right) \tilde{v} / \phi\right] t
$$


assuming that $\xi=H$ at $t=0$. We thus recover a diffusion equation for the drying front with an effective diffusion coefficient $D_{e}=2 D_{P}\left(c_{P}^{\text {sat }}-c_{P}^{*}\right) \tilde{v} / \phi$. We define the final drying time such as $\xi\left(t_{f}\right)=0$, leading to $t_{f}=H^{2} / D_{e}$, which leads to a more compact writing: $[H-\xi(t)]^{2}=t / t_{f}$. It is worth mentioning that this quasi-static approach is not universal and requires the wet-to-dry interface to move slowly as compared to the time it takes for the solubilized water concentration field to equilibrate to a linear profile by diffusion in the dry zone [19]. The argument is even made stricter with $t_{f} \gg H^{2} / D_{P}$, which eventually leads to $\phi \gg c_{P}^{\text {sat }} \tilde{v}$. With $c_{P}^{\text {sat }} \approx 40 \mathrm{~mol}^{-3} \mathrm{~m}^{-3}$, our quasistatic approximation remains valid for $\phi \gg 10^{-3}$, in line with our experiments where $\phi>210^{-2}$. Translating the front kinetics into the relative mass loss, we obtain a simple law: $W=1-\left(t / t_{f}\right)^{1 / 2}$, in fair agreement with experiments, and the prediction:

$$
t_{f}=\phi H^{2} /\left[2 D_{P}\left(c_{P}^{\mathrm{sat}}-c_{P}^{*}\right) \tilde{v}\right]
$$

which is also compatible with experiments, see Fig. 2. Indeed, we get $t_{f}=\alpha \phi$ with $\alpha=(2.2 \pm 0.1) 10^{5} \mathrm{~s}$ which yields $D_{P}\left(c_{P}^{\text {sat }}-c_{P}^{*}\right) \tilde{v}=(2.3 \pm 0.1) 10^{-12} \mathrm{~m}^{2} \cdot \mathrm{s}^{-1}$, and $t_{f}=\beta H^{2}$ with $\beta=(22 \pm 4) 10^{9} \mathrm{~s} . \mathrm{m}^{-2}$ which yields $D_{P}\left(c_{P}^{\text {sat }}-c_{P}^{*}\right) \tilde{v}=(6.8 \pm 0.5) 10^{-12} \mathrm{~m}^{2} \cdot \mathrm{s}^{-1}$. The two values agree reasonably well considering the different experimental uncertainties and the approximations made in this model. It is quite unfortunate that the solubility and mobility of water in this precise formulation of PDMS at $60^{\circ} \mathrm{C}$ is unknown. However, transposing to values extracted from literature at room temperature in another PDMS [20], we get $D_{P} c_{P}^{\text {sat }} \tilde{v} \approx 710^{-13} \mathrm{~m}^{2} \cdot \mathrm{s}^{-1}$. We believe the agreement is satisfying with our value because a higher temperature should imply a higher solubility along with a larger mobility of water molecules. 


\subsection{Supercritical drying}

An alternative method to avoid the collapse of the materials upon drying one consists in eliminating the existence of the water/gas interface during drying by using supercritical $\mathrm{CO}_{2}$ as performed by Martina et $a$ l. [21]. This technique is commonly used for the drying of silica to obtain aerogels [11]. However, since liquid $\mathrm{CO}_{2}$ is not miscible with water, water is progressively exchanged with ethanol by plunging the wet material in a water bath and slowly enriching the medium with liquid ethanol using a peristaltic pump. At the end of this exchange process, one obtains ethanol droplets embedded in the PDMS matrix. The material is then introduced in a tank reactor in which the pressure and temperature can be adjusted. The reactor is then slowly pressurized with $\mathrm{CO}_{2}$ at a constant flow rate of $11 \mathrm{~g} / \mathrm{min}$ and the pressure and temperature are adjusted to $P=11 \mathrm{MPa}$ and $T=50^{\circ} \mathrm{C}$ and kept constant during the drying process. $\mathrm{CO}_{2}$ mixes with ethanol resulting in a single supercritical phase in these conditions. Pure $\mathrm{CO}_{2}$ being constantly introduced into the reactor, the ethanol is slowly extracted, and the system finally only contains supercritical $\mathrm{CO}_{2}$. The pressure is then slowly decreased to $0.1 \mathrm{MPa}$ (over 1 hour) and the temperature is decreased to ambient conditions. Thus, the system never crosses any phase transition lines and no liquid-gas interface is present in the system.

\section{3. $\mathrm{H}_{2} \mathrm{O}_{2}$-assisted drying}

The third method consists of introducing a proportion of hydrogen peroxide $\left(\mathrm{H}_{2} \mathrm{O}_{2}\right)$ in the dispersed water phase [22]. $\mathrm{H}_{2} \mathrm{O}_{2}$ is spontaneously decomposed in $\mathrm{H}_{2} \mathrm{O}$ and $\mathrm{O}_{2}$ (gas) and the nucleation of the gas bubbles inside the pores allows for a release of the negative pressure endured by the material during the drying process. As a consequence, as drying occurs, one expects the nucleated gas bubbles to expand and replace the liquid that permeates through the polymer matrix. However, the decomposition reaction of hydrogen peroxide in oxygen and water has a very low rate constant at room temperature and in the absence of any catalyst. As a consequence, the decomposition of $\mathrm{H}_{2} \mathrm{O}_{2}$ does not occur during the emulsification process and only starts during the enlightening of the material with the UV lamp and the following drying step that is completed at $80^{\circ} \mathrm{C}$. Indeed, kinetic measurements of oxygen production 
(not shown here) show that the rate constant of the reaction increases by more than one order of magnitude when increasing the temperature from $20^{\circ} \mathrm{C}$ to $80^{\circ} \mathrm{C}$.

To observe the drying process, , the samples were UV polymerized and then rapidly put in a thermally controlled Peltier microscope cell at a drying temperature of $80^{\circ} \mathrm{C}$ and observed at different times. Due to the difference in refractive indices of PDMS, water, and air, the drying phase fronts are visible, and the change in the drying front can be clearly observed. We performed these observations for both the $\mathrm{OH} 457-\mathrm{H}_{2} \mathrm{O}$ and $\mathrm{OH} 457-\mathrm{H}_{2} \mathrm{O}_{2}$ systems. The optical microscopy pictures obtained at different times after the UV polymerization in these two cases are presented on Fig. 4.

At $t=0$, the images were taken just after the sample's introduction in the Peltier cell at a temperature of $80{ }^{\circ} \mathrm{C}$. Initially, both samples appear very dark, which is due to the strong scattering of light induced by the difference of optical index between the water that is contained inside the droplets and the continuous PDMS phase. As time passes, a drying front is observed between a semi-transparent region that appears at the edge of the sample and moves towards the center. The red arrow shows the direction of propagation of this drying front. The undried region appears dark due to the light scattering by water-filled pores.

In both samples (and up to $t=10 \mathrm{~min}$ ), the presence of the semi-transparent region indicates that the system becomes less scattering, which is due to the collapse of the pores as the water phase escapes the material. At $t=10$ min, a difference of behavior is observed between the two systems: in the $\mathrm{OH} 457-\mathrm{H}_{2} \mathrm{O}_{2}$ system, a dark zone starts to appear again at the edge of the sample, which indicates the reappearance of a strong scattering of light by the sample. This means that upon drying pores opens again in the first system but not in the case of the $\mathrm{OH} 457-\mathrm{H}_{2} \mathrm{O}$, which still remains almost transparent up to 35 minutes after the beginning of the experiment. By contrast the $\mathrm{OH} 457-\mathrm{H}_{2} \mathrm{O}_{2}$ system remains completely dark, which is due to the presence of air inside the pores. This means that, after a given amount of time (about 10 minutes in our case), the nucleation of $\mathrm{O}_{2}$ gas bubbles occurs in the system, 
which leads to a release of this internal stress that applies to the pores during drying and the pores reopen to reach back their initial size and shape.

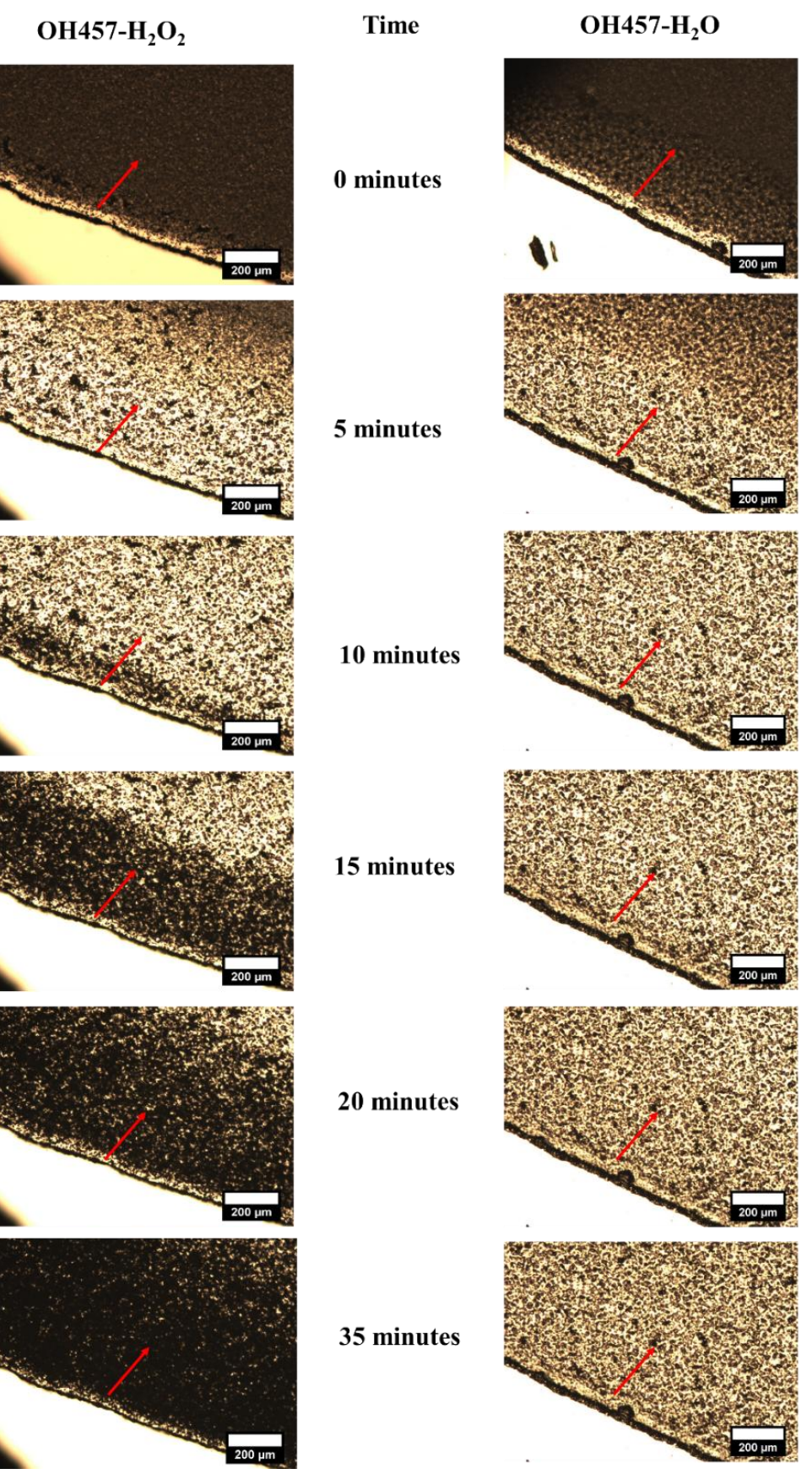

Figure 4: In-situ optical microscopy observations of the drying kinetics of cross-linked emulsions stabilized with $\mathrm{OH} 457$ containing (left column) or not (right column) $\mathrm{H}_{2} \mathrm{O}_{2}$ in the dispersed phase. The samples are maintained at $T=80^{\circ} \mathrm{C}$, squeezed between two glass slides and drying occurs from the border of the sample towards its center following the direction of the red arrow. 
Such method allows the system to be simply dried at room pressure while avoiding the step of exchange of water with ethanol. To achieve the targeted porosity, we optimized the concentration of $\mathrm{H}_{2} \mathrm{O}_{2}$ and found out the concentration of $15 \%$ to be the optimal one to achieve the targeted porosity.

\section{Characterization of the porous samples}

The microstructures of obtained dried porous samples with the two above methods were again characterized by SEM. Fig. $5(A)$ and (B) show the images of dried porous samples obtained with the $\mathrm{H}_{2} \mathrm{O}_{2}$-assisted (A) and the supercritical drying (B) techniques. The images are very similar and exhibit, in both cases, perfectly circular pores with a size distribution (Fig. 5 (C)) very close to the one of the initial emulsions. The sizes were obtained using ImageJ software for the analysis of the SEM pictures, while static light scattering experiment was performed to characterize the initial emulsion droplets size distribution. The porosity of the samples performed with the three drying techniques were then measured using a home-made setup measuring the Archimedes force acting on samples immerged in a water-glycerol mixture. The obtained values were more precise than the ones obtained by direct calculation from the mass and the dimensions of the samples. Fig. 5 (D) shows the final porosity (\%) as a function of the dispersed water phase volume fraction (\%) in emulsion for the three drying techniques. In the case of regular drying, a large difference between the final porosity of dried sample (that never exceeds $5 \%$ whatever the initial water volume fraction in the emulsion) and the dispersed water phase volume fraction in the initial emulsion is measured, which is a direct consequence of the pores collapse during drying, as observed in a previous study [10]. In contrary, supercritical and $\mathrm{H}_{2} \mathrm{O}_{2}$-assisted drying allow for a remarkable control over the samples porosity as evidenced by the data of Fig. 5 (D) where the porosity is roughly equal to the initial water volume fraction.

To determine the surface area of the samples, they were also all submitted to nitrogen adsorption measurements. The BET analysis of our samples (not shown here) led to very low values of the specific surface area, i.e. less than 0.5 $\mathrm{m}^{2} / \mathrm{g}$ for all our samples, which is the detection limit of the apparatus. This means that these samples do not exhibit any microporosity (pores in the nanometer range). Thus, a rough calculation of the expected surface area for such 
samples can be made by considering the contribution from the macropores that were observed using SEM. In this case, the surface specific area $S_{S}$ of a sample of volume $V_{\text {tot }}$, porosity $\phi$ and mass density $\rho$ should be equal to:

$$
S_{S}=\frac{\frac{\phi V_{\text {tot }}}{V_{\text {pore }}} S_{\text {pore }}}{\rho V_{\text {tot }}}=\frac{3 \phi}{\rho R}
$$

Where $V_{\text {pore }}=4 / 3 \pi R^{3}$ and $S_{\text {pore }}=4 \pi R^{2}$ are the volume and the surface of one pore of radius $R$, respectively. For the samples with the highest porosity $(\phi=30 \%$ ) and having a radius $R \sim 2.5 \mu \mathrm{m}$ (see Fig 5C) and a density $\rho$ of around $700 \mathrm{~kg} / \mathrm{m}^{3}$, we thus obtain a very low specific surface area $S_{\mathrm{s}} \sim 0.5 \mathrm{~m}^{2} / \mathrm{g}$, in good agreement with the BET results.
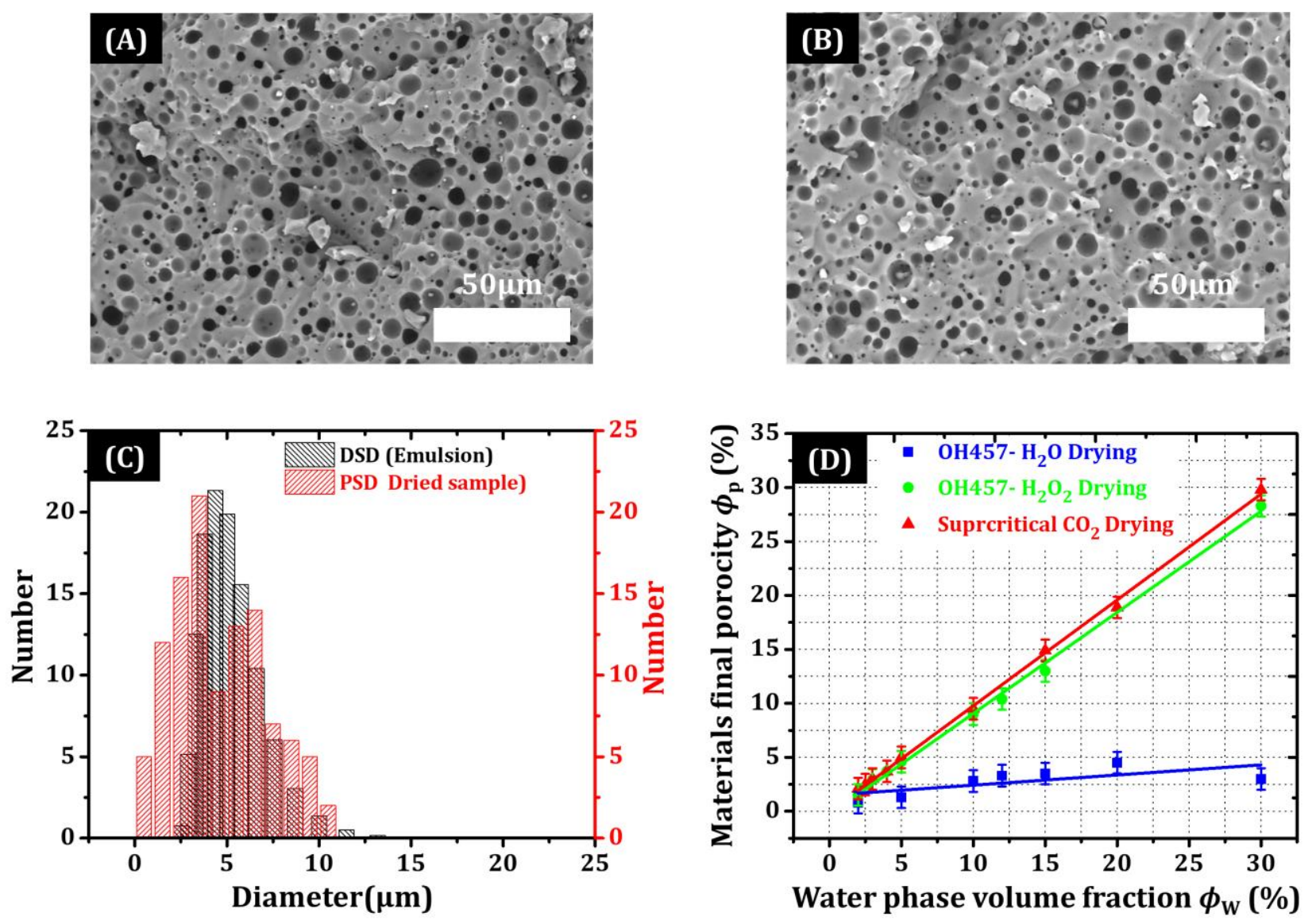

Figure 5: Characteristics of the final porous materials: (A-B) SEM pictures of the final materials obtained with $(A) \mathrm{H}_{2} \mathrm{O}_{2}$-assisted drying (B) supercritical drying ; (C): Pore size distributions(PSD) obtained from the SEM pictures for supercritical drying (the same is obtained for $\mathrm{H}_{2} \mathrm{O}_{2}$-assisted drying, not shown here) and water droplet size distribution (DSD) from static light scattering experiment on the initial emulsions; (D): evolution of the materials final porosity as a function of initial water volume fraction of the emulsions for the three drying methods. The solid lines on (D) are simple guides to the eyes. 


\section{Acoustic properties}

\subsection{Acoustic properties of the bulk materials}

The experimental device used to measure the acoustic velocity in the samples consists of two identical piezoelectric transducers with diameters $30 \mathrm{~mm}$ (Olympus 301). One transducer serves as a source and the other one as a receiver. These are placed face to face on a manual device, graduated, linearly sliding for varying the distance between the transducers, thereby enabling an accurate measurement of the sample thickness. The transducers are broadband with a central frequency of $500 \mathrm{kHz}$. For each given porosity, the measurements are performed on two samples with thicknesses of 2 and $3 \mathrm{~mm}$ (with a constant diameter of $32 \mathrm{~mm}$ ). Using a pulse generator (Olympus 5077PR-40-E), the transmitting transducer is excited by an electrical pulse of amplitude 400V with a repetition rate of $100 \mathrm{~Hz}$ allowing thereby the generation of a wideband acoustic signal. The acoustic signal transmitted and detected by the receiver is displayed on an oscilloscope (type Lecroy 9450A). The thickness of the sample is equivalent to the wave propagation distance. Because of the high dissipation of waves in the sample that is due to the macroporous nature of the materials, the excitation signals with frequencies centered around $500 \mathrm{kHz}$ are totally absorbed before reaching the receiver. So, the receiver thus only detects the waves of lowest frequencies (normally 20 to $200 \mathrm{kHz}$ ), which are less dissipated than the one of highest frequency.

We then performed the acoustic characterizations of the obtained samples and measured the evolution of the longitudinal phase velocity $\left(C_{\mathrm{L}}\right)$ as a function of the materials porosity (Fig. 6). From this curve, the evolution of the acoustic refraction index $(n)$ as a function of porosity $(\phi)$, was deduced as ratio of phase velocities in porous material and water $\left(n=C_{\mathrm{L} \text {, material }} / C_{\mathrm{L} \text {, water }}\right)$. As in previous papers $[17,23]$, we compared our data to theoretical predictions [24], expecting the following equation to describe the behavior of $C_{\mathrm{L}}$ as a function of porosity $\phi$ :

$$
C_{L}(\phi) \approx \frac{C_{L}(0)}{\sqrt{1+\frac{3 K_{0}}{4 G_{0}} \phi}}
$$


Where $C_{\mathrm{L}}(0), K_{0}$ and $G_{0}$ are, respectively, the sound speed $(\approx 1100 \mathrm{~m} / \mathrm{s})$ and the bulk isotropic and shear moduli of the pure non porous matrix. We measured $G_{0} \approx 3+/-1 \mathrm{MPa}$ and estimate $K_{0}=\rho_{0} C_{\mathrm{L}}(0)^{2} \approx 1000 \mathrm{MPa}$, where $\rho_{0}$ is the mass density of the pure matrix $\left(\approx 10^{3} \mathrm{~kg} / \mathrm{m}^{3}\right)$. Using such values and equation (7), we get the data plotted on Fig. 6 , in reasonable agreement with our experimental results.

It clearly comes out that the velocity only depends on the material's final porosity whatever the used technique of drying. The curve also shows that porosity larger than $5 \%$ cannot be obtained using classical drying due to the partial collapse of the material. From these studies, we deduce that porous materials with very well controlled porosity and consequently very well-defined acoustic index can be obtained. In the next section, we use these materials to fabricate an acoustic device, as an example of what can be achieved with these materials.

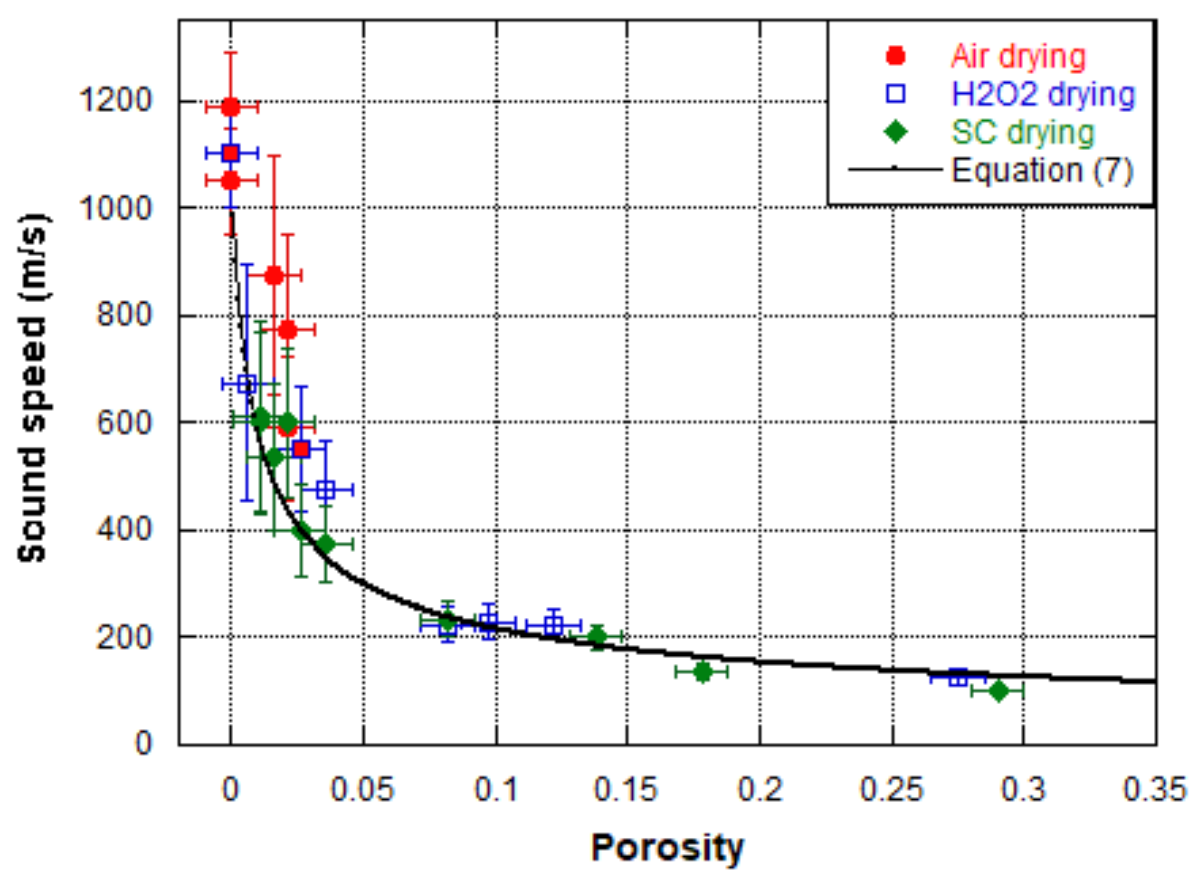

Figure 6: Evolution of the phase velocity as a function of the porosity at a fixed frequency of $90 \mathrm{kHz}$ for the materials prepared with the three different drying methods. Solid line: Calculation of the acoustic velocity using equation (7) in the text with $K_{0}=1000 \mathrm{MPa}$ and $G_{0}=3 \mathrm{MPa}$. 


\subsection{Fabrication of the final GRIN samples}

In a recent paper, we showed that such well-defined porous materials can be assembled to obtain gradient index (GRIN) acoustic metasurfaces [9]. In this section, we briefly show how the samples were technically assembled and present one example of acoustic device as wave deflector.

On Fig. 7 (B), we present the sample that was used for beam deflection, which requires a linear gradient index along the $X$ direction:

$$
n(X)=n(X=0)+\frac{\sin (\theta) X}{d}
$$

where $X$ indicates the axis of the porosity gradient, $\theta=5$ degrees is the chosen deflecting angle and $d=2 \mathrm{~mm}$ is the sample thickness, We define the width of each stripe (interval in $X$ ) as $10 \mathrm{~mm}$. Based on the results plotted on fig.6, the porosity of each stripe is then determined.

The GRIN sample was obtained using the procedure described in fig.7 (A), by layer by layer deposition of emulsions having required initial water volume fraction. Thanks to the immediate UV polymerization of each layer, no mixing of the emulsions was observed, and the porosity domains are very well separated. 

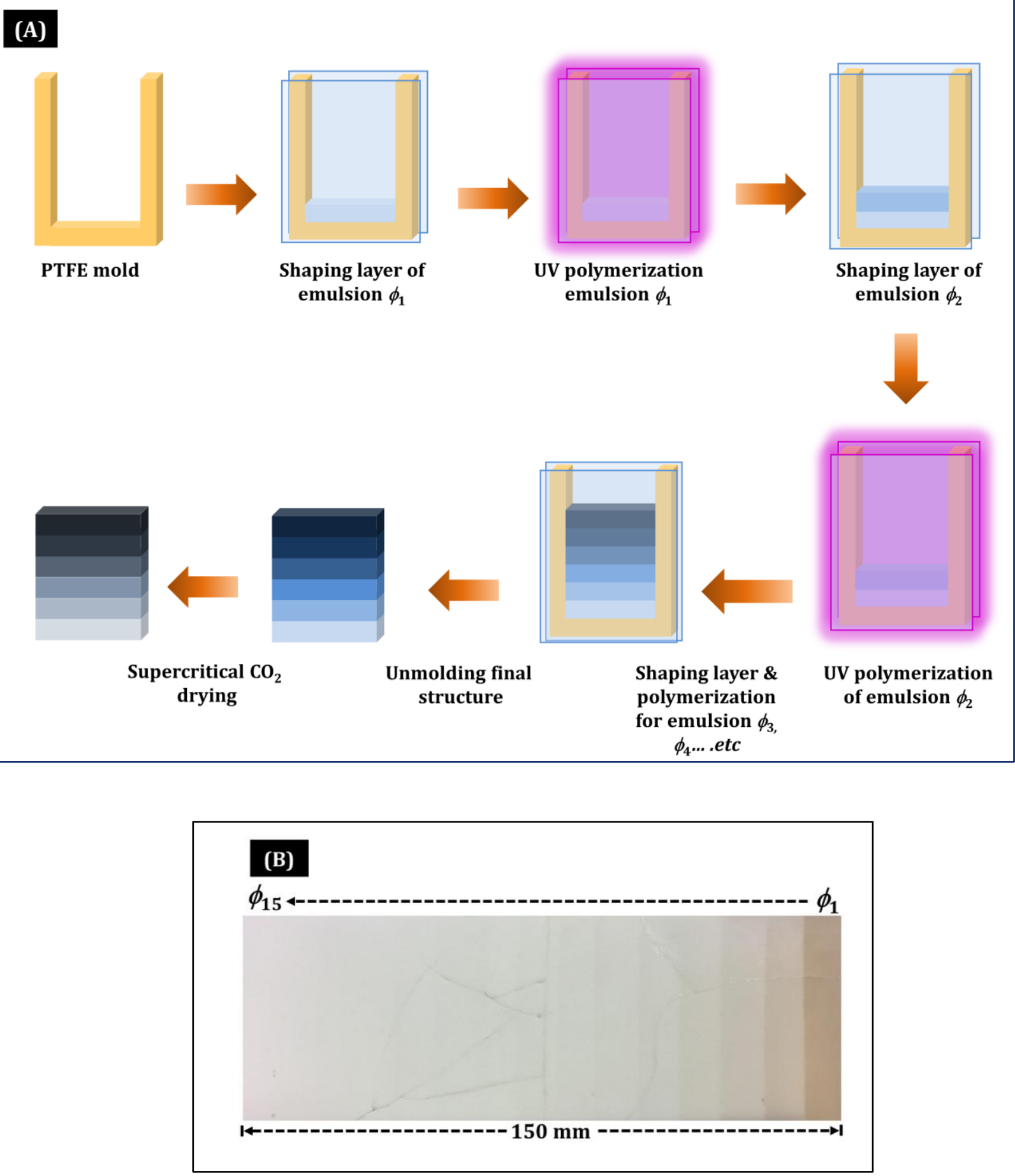

Figure 7: (A) Schematic of the procedure used for the fabrication of GRIN metasurfaces. From top to bottom: a thin polymer mold (nylon of polylactic acid (PLA) for example) is fabricated using a 3D printer. Emulsions with chosen volume fractions are introduced in each compartment and the system is then polymerized and dried (using supercritical CO2). (B) The final material is obtained and exhibit acoustic indices, which are respectively for each stripe (from right to left in the picture): $2.18 ; 2.69 ; 3.06 ; 3.61 ; 4.16 ; 4.41$;

$4.69 ; 5.11 ; 5.68 ; 6.25 ; 6.53 ; 6.81 ; 7.04 ; 7.27 ; 7.5$ 


\subsection{Acoustic properties of the GRIN metasurface}

To evidence the ability of our metasurface in terms of wavefront shaping, ultrasonic experiments were carried out in a water tank. A large planar ultrasonic (US) transducer (150 mm x $40 \mathrm{~mm}$ ) was used to generate plane waves propagating along the Z-axis as shown in Fig.8 (left). The central frequency $f$ was here of $150 \mathrm{kHz}$ corresponding to an incident wavelength $\lambda_{0}$ of $10 \mathrm{~mm}$ for these water-borne waves. The acoustic pressure field was then scanned in the $X Z$-plane thanks to a tiny hydrophone ( $1 \mathrm{~mm}$ in diameter) mounted on motorized linear stages.

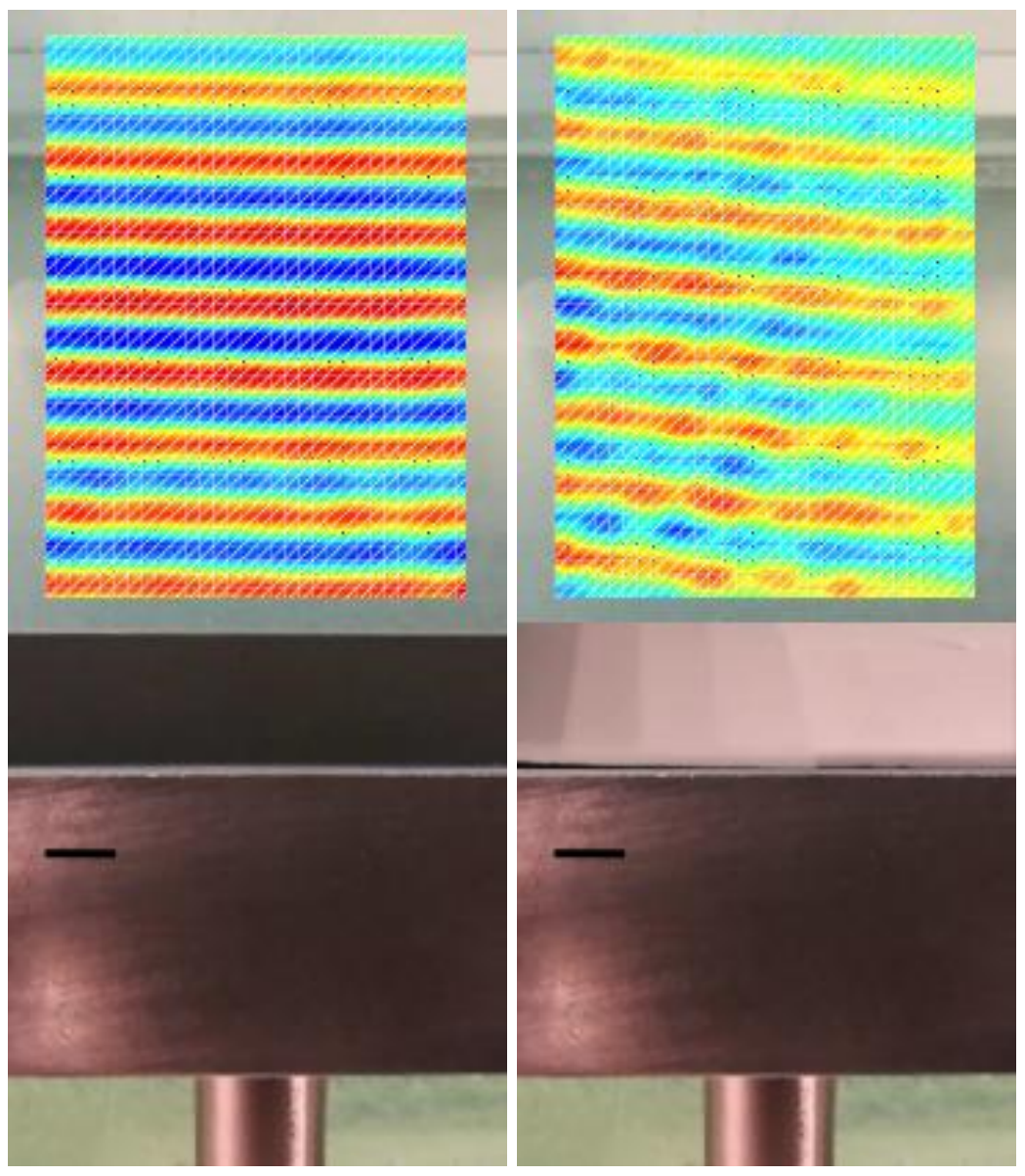

Figure 8: Measured pressure fields at $150 \mathrm{kHz}$ coming from a large flat ultrasonic transducer with no sample (left), with the deflecting metasurface (right). The black bar indicates $1 \mathrm{~cm}$.

When the US transducer is covered by the linear GRIN metasurface, the wavefronts passing through this subwavelength coating are still planar but slightly tilted from the initially horizontal $X$-axis as shown in Fig. 8 (right). The 
measured angle of deflection is $5^{\circ}$ in good agreement with the theoretical design of the metasurface index-profile. These results inspire us to realize materials exhibiting spatial modulation of refractive index by modulating the porosity inside the materials. We explore this opportunity to the realization of the devices that are referred to as "soft acoustic gradient index metasurfaces", which can be used to focus, steer, or to create acoustic vortex beam. These results are discussed in detail elsewhere [9].

\section{Conclusion}

Using an emulsion templating method coupled to different drying techniques, we show that obtaining soft porous materials with closed cells geometry and controllable values of their porosity can only be obtained using specific drying techniques. The regular air drying of these materials with no specific precautions leads to collapsed materials, in agreement with previous results $[10,18]$ with uncontrolled values of their porosity. By contrast, and in agreement with results from the literature $[21,22]$, drying using supercritical $\mathrm{CO}_{2}$ or with systems containing $\mathrm{H}_{2} \mathrm{O}_{2}$ in the cells lead to materials with fully controlled porosity. In these two cases, we show that the material final porosity is equal to the initial water content in the emulsion.

When the samples are dried in atmospheric conditions (regular drying), our data show that the drying kinetics obeys

a simple law of the type: $1-\left(\frac{t}{t_{F}}\right)^{1 / 2}$ in good agreement with the model that is developed in this paper. The required drying time is also shown to scale as the square of the sample thickness and linearly with the initial water volume fraction, which is also depicted by our model.

Using an adapted ultrasound acoustic setup, we show that the longitudinal wave acoustic velocity in the porous samples, and consequently the materials acoustic index, depend critically on their porosity, following a wellestablished model [24]. By a spatial control of the porosity distribution, along the lateral dimensions of the samples, we show that it is possible to obtain gradient index acoustic metasurfaces allowing acoustic wavefront shaping while being much thinner than the incident wavelength. The procedure is simple and easily up-scalable, adaptable to any 
types of acoustic transducers and exhibits a unique versatility in terms of wavefront shaping. The obtained devices are broadband and active for acoustic waves travelling in water.

\section{Acknowledgements}

We thank Frédéric Marchal and Tania Ireland from Elkem Company for fruitful discussions and for providing us with silicone rubber. Special thanks to Marco Faustini and Philippe Barois for fruitful suggestions and discussions. Special thanks to Marie-Anne Dourges, from Institut des Sciences Moléculaires - CNRS - Univ. of Bordeaux, for her help on BET analysis. This work was partially funded and performed within the framework of the Labex AMADEUS ANR-10LABEX-0042-AMADEUS with the help of the French state Initiative d'Excellence IdEx ANR-10-IDEX-003-02 and project BRENNUS ANR-15-CE08-0024.

\section{References}

[1] Xu, Y.; Fu, Y.; Chen, H. Planar Gradient Metamaterials. Nat. Rev. Mater. 2016 1(12), 16067

[2] Assouar, B.; Liang, B.; Wu, Y.; Li, Y.; Cheng, J.C. ; Jing, Y. Acoustic metasurfaces. Nat. Rev. Mater. 2018 3,460-472

[3] Xie, Y.; Wang, W.; Chen, H.; Konneker, A.; Popa, B.I.; S. A. Cummer, S.A. Wavefront modulation and subwavelength diffractive acoustics with an acoustic metasurface. Nat. Commun. 2014 5, 5553

[4] Li, Y.; Yu, G.; Liang, B.; Zou, X.; Li, G.; Cheng, S.; Cheng, J. Three-dimensional Ultrathin Planar Lenses by Acoustic Metamaterials. Sci. Rep. 2014 4, 6830

[5] Xie, B.; Tang, K.; Cheng, H.; Liu, Z.; Chen, S.; Tian, J. Coding Acoustic Metasurfaces. Adv. Mater. 2017 29, 1603507

[6] Tang, H.; Chen, Z.; Tang, N.; Li, S.; Shen, Y.; Peng, Y.; Zhu, X.; Zang, J. Hollow-Out Patterning Ultrathin Acoustic Metasurfaces for Multifunctionalities Using Soft fiber/Rigid Bead Networks. Adv. Funct. Mater. 201828 (36), 1801127

[7] Su, X.; Norris, A.N.; Cushing, C.W.; Haberman, M.R.; Wilson, P.S.; Broadband focusing of underwater sound using a transparent pentamode lens. J. Acoust. Soc. Am. 2017141 (6) 4408-4417 
[8] Calvo, D.C.; Thangawng, A.L.; Nicholas, M.; Layman, C.N. Thin Fresnel zone plate lenses for focusing underwater sound. Appl. Phys. Lett. 2015107 (1), 014103

[9] Jin, Y.; Kumar, R.; Poncelet, O.; Mondain-Monval, O.; Brunet, T. Flat acoustics with soft gradient-index Metasurfaces. Nat. Commun. 2019 10, 143

[10] Kovalenko, A.; Zimny, K.; Mascaro, B.; Brunet, T.; Mondain-Monval, O. Tailoring of the porous structure of soft emulsion-templated polymer materials. Soft matter 2016 12, 5154-5163.

[11] Bisson, A.; Rigacci, A.; Lecomte, D.; Rodier, E.; Achard, P. Drying of Silica Gels to Obtain Aerogels: Phenomenology and Basic Techniques. Drying Technology 2003 21(4), 593-628

[12] Lumelsky, Y.; Silverstein, M.S. Biodegradable Porous Polymers through Emulsion Templating. Macromolecules $200942(5), 1627-1623$

[13] Wheeler, T.D.; Stroock, A.D. The transpiration of water at negative pressures in a synthetic tree. Nature 2008 455 (7210), 208-212

[14] Silverstein, M.S. PolyHIPEs: Recent advances in emulsion-templated porous polymers. Prog. Polym. Sci. 201439 (1), 199-234

[15] Pulko, I.; Krajnc, P. High Internal Phase Emulsion Templating - A Path To Hierarchically Porous Functional Polymers. Macromol. Rapid Commun. 201233 (20), 1731-1746

[16] Zimny, K.; Merlin, A.; Ba, A.; Aristégui, C.; Brunet, T.; Mondain-Monval, O. Soft Porous Silicone Rubbers as Key Elements for the Realization of Acoustic Metamaterials. Langmuir 2015 31, 3215-3221

[17] Kovalenko, A.; Fauquignon, M.; Brunet T.; Mondain-Monval, O. Tuning the sound speed in macroporous polymers with a hard or soft matrix. Soft Matter 2017 13, 4526-4532;

[18] Milner, M.P.; Jin, L.; Hutchens, S.B. Creasing in evaporation-driven cavity collapse. Soft Matter 2017, 13, 6894 [19] Daubersies L.; Salmon, J.B. Evaporation of solutions and colloidal dispersions in confined droplets. Phys. Rev. E 2011, 84, 031406 
[20] Dollet, B.; Louf, J.F.; Alonzo, M.; Jensen, K.H.; Marmottant, P. Drying of channels by evaporation through a permeable medium. J. R. Soc. Interface 2019, 16 (151), 20180690

[21] Della Martina, A.; Hilborn, J.G.; Kiefer, J.; Hedrick, J.L.; Srinivasan, S.; Miller, R.D. Siloxane Elastomer Foams. In Polymeric Foams: Science and Technology, Vol. 669, ACS Symposium Series (Ed. K. C. Khemani) Ch. 2, 8-25 (American Chemical Society, Washington DC, 1997)

[22] Bae, S.E.; Son, J.S.; Park, K.; Han, D.K. Fabrication of covered porous PLGA microspheres using hydrogen peroxide for controlled drug delivery and regenerative medicine. J. Controlled Release 2009133 (1), 37-43

[23] Ba, A.; Kovalenko, A.; Aristégui, C.; Mondain-Monval, O.; Brunet, T. Soft porous silicone rubbers with ultra-low sound speeds in acoustic metamaterials. Scientific Reports 2017 7, 40106

[24] Kuster, G.; Toksöz, M. Velocity and attenuation of seismic waves in two-phase: Part I. Theoretical formulations. Geophysics 1974, 39 (5), 587-606. 


\section{TOC Graphic}

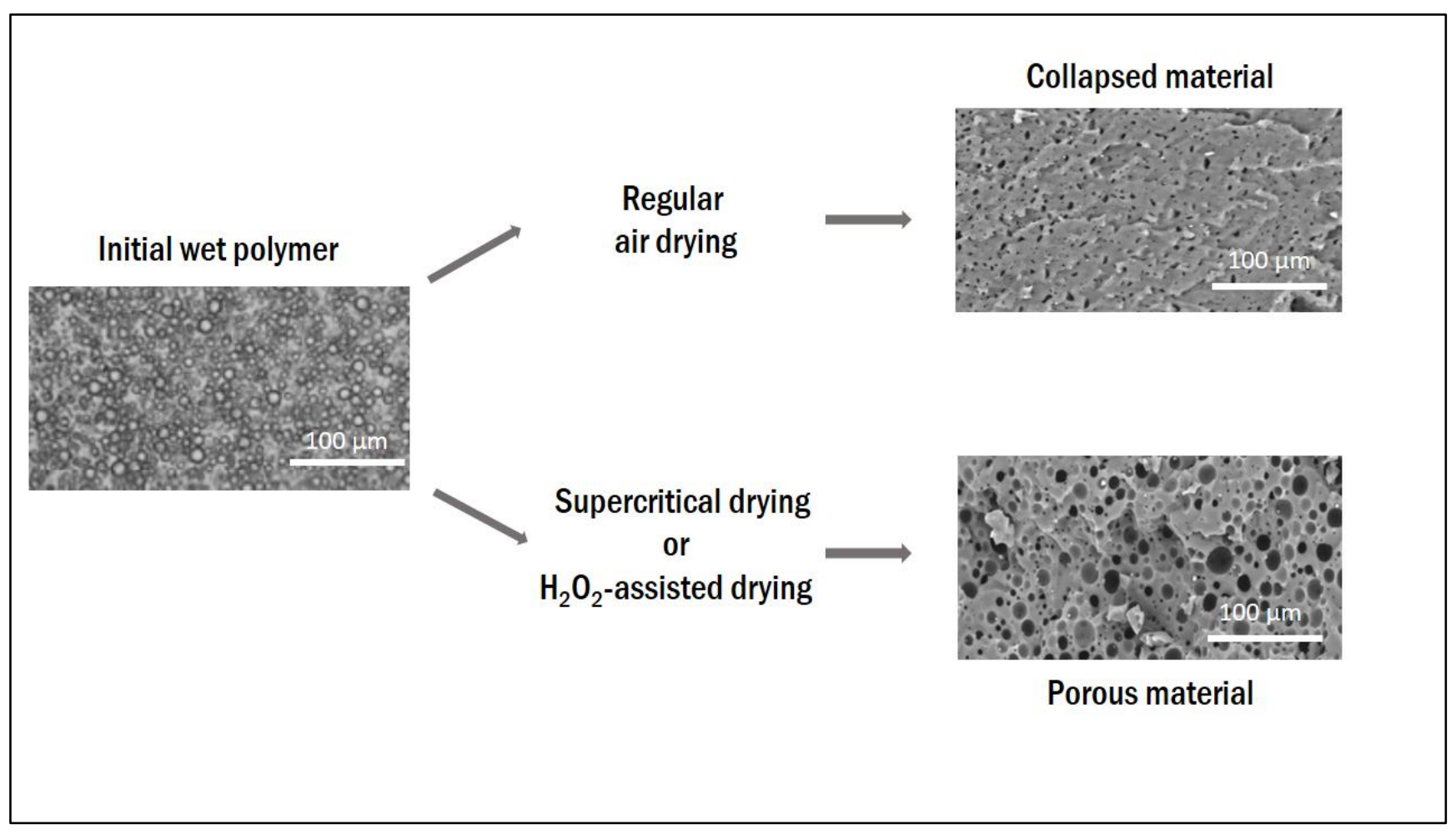

